\title{
Difference in inflammation, atherosclerosis, and platelet activation between coronary artery aneurysm and coronary artery ectasia
}

\author{
Wei Wei ${ }^{1 \#}$, Xingxu Wang ${ }^{1 \#}$, Zhenghao Huang ${ }^{1}, \mathrm{Xiaolin}^{\mathrm{Li}}{ }^{2}, \mathrm{Yu} \mathrm{Luo}{ }^{1 \wedge}$ \\ ${ }^{1}$ Department of Cardiovascular Medicine, East Hospital, Tongji University School of Medicine, Shanghai, China; ${ }^{2}$ Department of Cardiovascular \\ Medicine, Jian East Hospital, Jinggangshan University School of Medicine, Jiangxi, China \\ Contributions: (I) Conception and design: W Wei, Y Luo; (II) Administrative support: W Wei, Y Luo; (III) Provision of study materials or patients: W \\ Wei, X Wang, Z Huang, Y Luo; (IV) Collection and assembly of data: W Wei, X Wang; (V) Data analysis and interpretation: W Wei, X Wang; (VI) \\ Manuscript writing: All authors; (VII) Final approval of manuscript: All authors. \\ \#These authors contributed equally to this work. \\ Correspondence to: Yu Luo. Department of Cardiovascular Medicine, East Hospital, Tongji University School of Medicine, 150 Jimo Road, Shanghai \\ 200120, China. Email: Wangyily1839@126.com; 14006394@qq.com.
}

Background: Coronary artery aneurysm (CAA) and coronary artery ectasia (CAE) may be two different types of coronary artery dilatation with unknown etiology. This study aimed to compare the differences between CAA and CAE and to investigate their pathogenesis and the necessity of antiplatelet therapy.

Methods: One hundred patients each with confirmed CAA, CAE, and normal coronary artery (NCA) from September 2017 to July 2019 were included. All patients completed examinations of the ankle-brachial index (ABI), pulse wave rate, and carotid ultrasonography; and were tested for routine blood, lipid, and immune parameters. Blood samples were collected 1 week after the withdrawal of antiplatelet drugs, and vascular inflammatory indexes, platelet activation indexes, thromboelastography, and the platelet aggregation rate were measured. Analysis of variance and the chi-square or Fisher exact test were used for statistical analysis.

Results: The perinuclear anti-neutrophil cytoplasmic antibody (ANCA), endothelial-1, matrix metalloproteinase-9, and tumor necrosis factor- $\alpha$ were significantly higher in CAE than in NCA, while cytoplasmic ANCA was appreciably higher in CAE than in CAA $(\mathrm{P}<0.05)$. Myeloperoxidase and growth/ differentiation factor-15 were significantly higher in CAE than in CAA and NCA $(\mathrm{P}<0.05)$. ABI was significantly lower in CAA and CAE than in NCA $(\mathrm{P}<0.05)$, low-density lipoprotein/high-density lipoprotein was significantly higher in CAA than in NCA $(\mathrm{P}<0.05)$, and the detection rate of carotid artery thickening was significantly higher in CAA than in CAE and NCA $(\mathrm{P}<0.05)$. The Gensini and SYNTAX scores were significantly higher in CAA than in CAE $(\mathrm{P}<0.05)$. The percentages of CD62P and PAC-1 were higher in CAA and CAE than in NCA $(\mathrm{P}<0.05)$. The arachidonic acid aggregation rate in CAA and adenosine 5 '-diphosphate aggregation rate in $\mathrm{CAE}$ were significantly higher than in $\mathrm{NCA}(\mathrm{P}<0.05)$. The values of thrombin formation time and reaction time were significantly lower in CAE than in NCA $(\mathrm{P}<0.05)$, and the $\alpha$ angle was significantly higher in CAE than in NCA.

Conclusions: CAE was closely related to inflammation, whereas CAA was closely related to atherosclerosis. Platelet activation was present in both diseases; therefore, antiplatelet therapy is recommended.

Keywords: Coronary artery ectasia (CAE); coronary artery aneurysm (CAA); inflammation; atherosclerosis; platelet activation

Submitted Apr 05, 2020. Accepted for publication Aug 21, 2020.

doi: $10.21037 /$ jtd-20-1579

View this article at: http://dx.doi.org/10.21037/jtd-20-1579

\footnotetext{
^ ORCID: 0000-0002-0046-5356.
} 


\section{Introduction}

Coronary artery dilation is a rare disease. Jarcho (1) first described the dilatation of coronary arteries in 1812 . Trinidad et al. (2) published a clinical study on coronary artery aneurysm (CAA) in 49 patients in 1953. Since then, the study of coronary artery dilation has become indepth. Because of the development and wide application of percutaneous coronary intervention and the popularity of coronary computed tomography angiography, the identification and diagnosis rate of coronary artery diseases has improved remarkably $(3,4)$. Since patients with such diseases are at risk of myocardial infarction, pericardial tamponade, and even sudden death, it is particularly important to understand their pathogenesis and clinical characteristics, and to treat them effectively.

Coronary artery dilatation has been confirmed for a long time, but its etiology and pathogenesis remain unclear. Until now, the nomenclature and classification of coronary artery dilatation have not been unified, especially for CAA and coronary artery ectasia (CAE), of which the application in such disease is confusing. Some studies have regarded CAA and CAE as two stages of coronary artery dilatation according to different symptoms (5), whereas others have indicated that the pathogenesis of CAA and CAE are different, but both are caused by coronary artery dilatation $(6,7)$. Increasingly, CAA and CAE are viewed as two different types of coronary artery dilatation (6-8).

We found that there were significant differences in coronary angiographic characteristics and cardiovascular risk factors between CAA and CAE. CAA may have a closer relationship with atherosclerosis (7); however, the specific pathogenesis of CAA and CAE needs further study. In addition, because anatomical structure, atherosclerosis, inflammatory activation, and other factors affect platelet function, there is no guideline or consensus on whether patients with different types of coronary dilatation need antiplatelet aggregation (i.e., antithrombotic therapy). Therefore, this study aimed to compare the differences between CAA and CAE in activation of the inflammatory system (including vasodilation, endothelial injury, inflammatory cytokines, and up-regulation of inflammatory cells), atherosclerosis indicators, and platelet activation, and to investigate their pathogenesis and the necessity of antiplatelet therapy. We present the following article in accordance with the STROBE reporting checklist (available at http://dx.doi.org/10.21037/jtd-20-1579).

\section{Methods}

\section{Study design and subjects}

This was an experimental basic study. Patients who were admitted to our hospital from September 2017 to July 2019 for coronary angiography were enrolled for selective coronary angiography using standard Judkins technology, and the results were evaluated by two experienced surgeons (9). All patients underwent examinations of the inflammatory system, atherosclerosis and platelet function. The exclusion criteria were as follows: (I) coronary artery dilatation occurred in or was directly related to the coronary artery bypass graft segment; (II) coronary artery dilatation appeared after percutaneous coronary intervention; (III) patients diagnosed with Kawasaki disease; (IV) coronary artery fistula or abnormality; (V) acute or chronic complete coronary artery occlusion; (VI) coronary artery dilatation appeared in the proximal segment of the coronary artery stenosis; (VII) cardiomyopathy, valvular heart disease, and congestive heart failure; (VIII) other diseases: coagulopathy, collagen tissue disease, vasculitis, syphilis, chronic obstructive pulmonary disease, pulmonary hypertension, early menopause, thyroid disease, liver and kidney failure, known malignant tumors, local or systemic infection, recent history of infection (3 months), and other inflammatory diseases; and (IX) anything that may interfere with the measurement of these markers, such as the use of oral anticoagulants, antiplatelet drugs, and glucocorticoids (administration of a routine oral loading dose of antiplatelet drugs was permitted before coronary angiography).

The study was approved by the Ethics Committee of Shanghai East Hospital Tongji University and conducted in line with the Declaration of Helsinki (as revised in 2013). All patients signed the written informed consent form.

Since September 2017, we continually enrolled 300 patients who met the diagnostic and exclusion criteria; each group included 100 patients. One hundred patients with coronary artery dilatation were included in the CAA group, i.e., those with a segment exceeding 1.5 times the diameter of an adjacent normal coronary artery (NCA) segment and involving blood vessels with a total length of less than $10 \mathrm{~mm}$. One hundred patients with coronary artery dilatation were included in the CAE group, i.e., those with a diameter of the dilated segment measuring more than 1.5 times that of the adjacent normal segment and the length of dilated lesions measuring more than $10 \mathrm{~mm}$. 
Lastly, 100 patients were included in the NCA group, i.e., those with normal coronary angiography findings (9). We recorded instances of coexistence with coronary stenosis for all of the patients in the CAA and CAE groups. The obstructive coronary artery lesions were evaluated by the Gensini index and the SYNTAX score.

\section{Study variables}

The following variables were compared among the CAA, CAE, and NCA groups: (I) differences in the activation status of the inflammatory system, including the peripheral blood cell count, high-sensitivity C-reactive protein (hsCRP), interleukin (IL)-6, cytoplasmic anti-neutrophil cytoplasmic antibody (c-ANCA), perinuclear anti-neutrophil cytoplasmic antibody (p-ANCA), endothelin-1 (ET-1), matrix metalloproteinase-9 (MMP-9), growth/differentiation factor 15 (GDF-15), myeloperoxidase (MPO), and tumor necrosis factor- $\alpha$ (TNF- $\alpha$ ); (II) differences in atherosclerosis, including blood lipid, ankle-brachial index (ABI), brachial-ankle pulse wave rate (baPWV), carotid ultrasonography findings, Gensini and SYNTAX scores; and (III) differences in platelet activation, including CD62P, procaspase-activating compound 1 (PAC-1), von Willebrand factor (vWF), adenosine 5 '-diphosphate (ADP) aggregation rate, arachidonic acid (AA) aggregation rate, and thromboelastography (TEG) indexes.

\section{Detection of inflammatory factors}

Before all patients were administered a routine oral loading dose of antiplatelet drugs before coronary angiography, $5 \mathrm{~mL}$ of intravenous ethylenediaminetetraacetic acidanticoagulated blood was collected after 8 hours of fasting; the sample was mixed upside down immediately and centrifuged at 2,000 rpm for 20 minutes after standing for 20 minutes. The separated plasma was placed into an Eppendorf tube, labeled, and stored at $-80^{\circ} \mathrm{C}$.

The UPPER automatic biochemical immunoprotein analyzer (Shanghai UPPER Bio-tech Pharma Co., Ltd., Shanghai, China) was used to measure hsCRP, and the COBAS E411 chemiluminometer (Roche Diagnostics, Basel, Switzerland) was used to measure IL-6. The indirect immunofluorescence method was used to detect c-ANCA and p-ANCA, and the kit was purchased from Oumeng Co., Ltd. (Art. No.: FA1201100513).

Blood samples were obtained at 1 week after the withdrawal of antiplatelet drugs. Other relevant inflammatory markers, including ET-1, MMP-9, GDF-15,
MPO, and TNF- $\alpha$, were detected using the enzyme-linked immunoassay (ELISA) method. The plasma ET-1 level detection, plasma MMP-9 level, and plasma GDF-15 level kits; the MPO content measurement kit; and the serum TNF- $\alpha$ level measurement kit were purchased from Abcam (Art. Nos.: ab2786, ab246539, ab155432, ab105136, and ab225576, respectively). The ELISA was performed as per the operation instructions provided by the reagent supplier.

\section{Measurement of baPWV and ABI}

$\mathrm{BaPWV}$ and $\mathrm{ABI}$ were measured using a Japanese Colin VP-1000 automatic arteriosclerosis tester. At room temperature (approximately $25^{\circ} \mathrm{C}$ ), patients were instructed to rest for 5 minutes before testing. The standard supine position was selected with patients' limbs restrained with oscillometric blood pressure cuffs, and the baPWV was recorded and analyzed simultaneously. The left and right baPWVs were measured once, and the higher value was used in the statistical analysis. Lateral ABI was defined as the systolic blood pressure of the ankle artery (posterior tibial artery or dorsalis pedis artery) divided by that of both brachial arteries. The left and right ratios were measured to obtain ABI values of the CAA and CAE groups, and the lower values were used as measured $A B I$ values in the statistical analysis.

\section{Carotid ultrasonography}

The HP 5500 ultrasound system (Philips Healthcare, Best, The Netherlands) was used, with a transducer frequency of 3-11 MHz. The examination was performed by a fulltime echocardiographer. Patients were required to lie in the supine position and move to the lateral head position to expose their carotid artery. The origin, trunk, and bifurcation of both carotid arteries should be measured routinely. The carotid intima-media thickness (IMT), peak systolic velocity (PSV), end-diastolic velocity (EDV), and resistance index (RI) were measured.

\section{Gensini and SYNTAX scores for digital coronary angiography}

The Gensini score was used in patients with coronary abnormalities. There was no need to perform the numerical score calculation in the NCA group. Combined with coronary angiography, the products of the piecewise integral and stenosis integral were added to the total integral. The 
Table 1 Comparison of baseline risk factors between the CAA and CAE groups

\begin{tabular}{lcccc}
\hline Risk factors & CAA $(\mathrm{n}=100)$ & $\mathrm{CAE}(\mathrm{n}=100)$ & $\mathrm{NCA}(\mathrm{n}=100)$ & $\mathrm{P}$ \\
\hline Age (years) & $65.6 \pm 11.3$ & $63.7 \pm 10.5$ & $61.0 \pm 11.3$ & 0.013 \\
Male sex (\%) & 70 & 75 & 53 & 0.003 \\
Diabetes (\%) & 26 & 24 & 17 & 0.276 \\
Smoking (\%) & 20 & 30 & 17 & 0.069 \\
Hyperlipidemia (\%) & 32 & 30 & 20 & 0.125 \\
Hypertension (\%) & 67 & 70 & 37 & 0.000 \\
Family history of CAD (\%) & 15 & 17 & 11 & 0.468 \\
\hline
\end{tabular}

CAA, coronary artery aneurysm; CAE, coronary artery ectasia; CAD, coronary artery disease.

integral of each patient was between 0 and 160 . The results were calculated by two cardiologists and averaged to score the angiographic lesions with coronary stenosis.

The SYNTAX score was calculated using the CVNET cardiovascular interventional management system V1.0 embedded with the SYNTAX scoring software (SN: SV0018, CREALIFE, Beijing, China). The lesion degree index was input to calculate the SYNTAX score of all lesion segments in each patient. The results were calculated by two cardiologists and averaged for score calculation.

\section{Detection of the platelet activation, platelet aggregation function, and TEG indexes}

Blood samples were obtained from all patients 1 week after the withdrawal of antiplatelet drugs, and CD62P and PAC-1 were measured using flow cytometry. The required reagents were purchased from the BD Company. The vWF was determined using the ELISA method, and the detection kit was purchased from Abcam (Art. No.: ab108918). The AggRAM platelet aggregation instrument (YuYan Instrument, Shanghai, China) was used to determine the platelet aggregation function, including the AA aggregation rate and $\mathrm{ADP}$ aggregation rate, after the turbidimetry method was used.

TEG was performed using the TEG 5000 coagulation monitoring system and its analyzer (Haemoscope Corporation, NY, USA). The detection of the following parameters was conducted automatically by the analyzer and computer software: reaction time $(\mathrm{R})$, thrombin formation time $(\mathrm{K})$, fibrin clot formation rate ( $\alpha$ angle), and blood clot strength (MA). TEG images and reference values were traced by the software.

\section{Statistical analysis}

SPSS statistical software, version 23.0 (IBM Corp., Armonk, NY, USA) was used to perform all data analyses. Continuous variables were tested using one-way analysis of variance (ANOVA). The mean expression (mean \pm standard deviation) and variance homogeneity test were performed first. For continuous variables with homogeneous variance, ANOVA was performed to test for significant differences, and the least significant difference test was used to perform pairwise comparisons; for continuous variables without homogeneous variance, the correction value derived from the Brown-Forsythe test (B) was used to indicate significant differences, and the Tamhane T2 test was used to perform pairwise comparisons. Categorical variables were compared using the chi-square or Fisher exact test. All tests were twotailed, and the statistical significance was defined as $\mathrm{P}<0.05$.

\section{Results}

\section{Comparison of baseline risk factors between the CAA and CAE groups}

Three groups were included in the study, each group contained 100 patients. As shown in Table 1 there are significant differences in age and sex between the CAA, $\mathrm{CAE}$ and NCA groups $(\mathrm{P}=0.013$ and $\mathrm{P}=0.003$, respectively). The proportion of men was higher in the CAE group (75\%) than in the other two groups. The prevalence rate of hypertension was higher in the CAA and CAE groups than that in the NCA group $(\mathrm{P}=0.000)$, but there were no significant differences in diabetes, smoking, hyperlipidemia, and family history of coronary heart disease (CAD) among the three groups. 
Table 2 Comparison of inflammatory cells in peripheral blood among the CAA, CAE, and NCA groups

\begin{tabular}{|c|c|c|c|c|c|c|c|}
\hline Inflammatory cells & CAA $(n=100)$ & CAE $(n=100)$ & $\operatorname{NCA}(n=100)$ & $\mathrm{P}$ & \multicolumn{3}{|c|}{$\mathrm{P}$ (pairwise comparison) } \\
\hline Neutrophil count $\left(\times 10^{9} / \mathrm{L}\right)$ & $4.52 \pm 1.93$ & $4.44 \pm 1.88$ & $4.48 \pm 1.95$ & 0.955 & NA & NA & NA \\
\hline Lymphocyte count (×109/L) & $1.71 \pm 0.59$ & $1.63 \pm 0.54$ & $1.50 \pm 0.56$ & 0.026 & 0.271 & 0.111 & 0.007 \\
\hline NLR & $3.12 \pm 2.45$ & $3.01 \pm 1.73$ & $4.06 \pm 5.77$ & 0.096 & NA & NA & NA \\
\hline Monocyte count $\left(\times 10^{9} / \mathrm{L}\right)$ & $0.50 \pm 0.21$ & $0.48 \pm 0.17$ & $0.46 \pm 0.24$ & 0.490 & NA & NA & NA \\
\hline Platelet count $\left(\times 10^{9} / \mathrm{L}\right)$ & $197.34 \pm 60.64$ & $207.09 \pm 46.4$ & $208.14 \pm 56.81$ & 0.311 & NA & NA & NA \\
\hline Mean platelet volume (fL) & $10.69 \pm 2.21$ & $10.37 \pm 1.40$ & $10.47 \pm 1.40$ & 0.34 & NA & NA & NA \\
\hline
\end{tabular}

CAA, coronary artery aneurysm; CAE, coronary artery ectasia; NLR, neutrophil-to-lymphocyte ratio; NCA, normal coronary artery; NA, not applicable.

Table 3 Comparison of inflammatory indexes among the CAA, CAE, and NCA groups

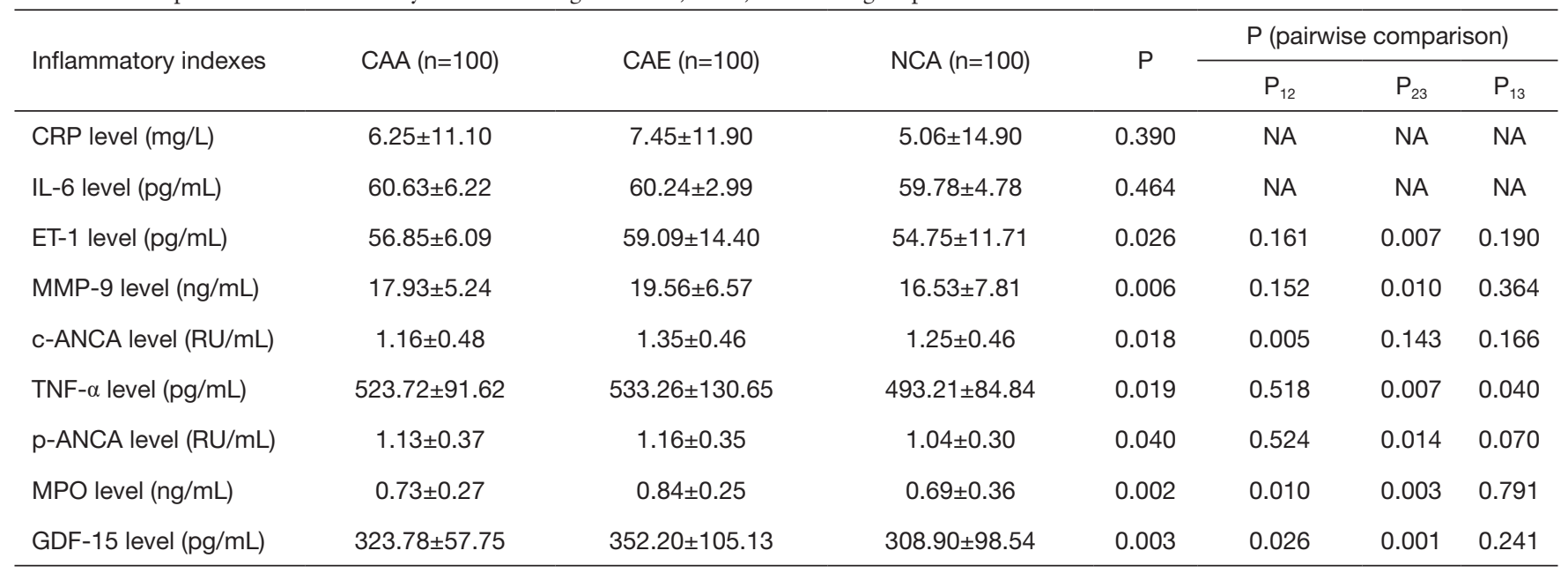

CAA, coronary artery aneurysm; CAE, coronary artery ectasia; NCA, normal coronary artery; CRP, C-reactive protein; IL-6, interleukin-6; ET-1, endothelin-1; MMP-9, matrix metalloproteinase-9; c-ANCA, cytoplasmic antineutrophil cytoplasmic antibody; TNF- $\alpha$, tumor necrosis factor- $\alpha$; p-ANCA, perinuclear anti-neutrophil cytoplasmic antibody; MPO, myeloperoxidase; GDF-15, growth/differentiation factor 15; NA, not applicable.

\section{Comparison of inflammatory cells in peripheral blood between the CAA and CAE groups}

Table 2 shows that the peripheral blood lymphocyte count is appreciably higher in the CAA group $\left[(1.71 \pm 0.59) \times 10^{9} / \mathrm{L}\right]$ than that in the NCA group $\left[(1.50 \pm 0.56) \times 10^{9} / \mathrm{L}\right]$, and the difference is statistically significant $(\mathrm{P}=0.007)$. However, there were no significant differences in total white blood cell count, mean neutrophil count, mean monocyte count, mean platelet count, mean platelet volume, and neutrophilto-lymphocyte ratio (NLR) among the CAA, CAE, and NCA groups.

\section{Comparison of inflammatory indexes between the CAA and CAE groups}

There were no significant differences in CRP and IL-6 levels among the three groups (Table 3). The p-ANCA ( $1.16 \pm 0.35$ vs. $1.04 \pm 0.30 \mathrm{RU} / \mathrm{mL}, \mathrm{P}=0.014)$, ET -1 ( $59.09 \pm 14.40$ vs. $54.75 \pm 11.71 \mathrm{pg} / \mathrm{mL}, \mathrm{P}=0.007)$, MMP-9 (19.56 \pm 6.57 vs. $16.53 \pm 7.81 \mathrm{ng} / \mathrm{mL}, \mathrm{P}=0.010)$, and TNF- $\alpha$ levels $(533.26 \pm 130.65$ vs. $493.21 \pm 84.84 \mathrm{pg} / \mathrm{mL}, \mathrm{P}=0.007)$ were significantly higher in the CAE group than in the NCA group. The c-ANCA level was significantly higher in the CAE group than in the CAA group $(1.35 \pm 0.46$ vs. 
Table 4 Comparison of atherosclerotic indexes atherosclerotic indexes among the CAA, CAE, and NCA groups

\begin{tabular}{|c|c|c|c|c|c|c|c|}
\hline Atherosclerotic indexes & CAA $(n=100)$ & $\operatorname{CAE}(n=100)$ & $\operatorname{NCA}(n=100)$ & $\mathrm{P}$ & \multicolumn{3}{|c|}{$\mathrm{P}$ (pairwise comparison) } \\
\hline baPWV (m/s) & $1,502.7 \pm 86.3$ & $1,472.3 \pm 143.7$ & $1,463.7 \pm 148.5$ & 0.083 & NA & NA & NA \\
\hline $\mathrm{ABI}$ & $0.78 \pm 0.19$ & $0.79 \pm 0.20$ & $0.85 \pm 0.18$ & 0.017 & 0.742 & 0.022 & 0.009 \\
\hline LDL/HDL & $2.73 \pm 1.29$ & $2.53 \pm 1.14$ & $2.33 \pm 0.83$ & 0.040 & 0.585 & 0.398 & 0.030 \\
\hline IMT (mm) & $0.89 \pm 0.38$ & $0.78 \pm 0.24$ & $0.76 \pm 0.37$ & 0.019 & 0.030 & 0.645 & 0.009 \\
\hline $\mathrm{PCV}(\mathrm{mm} / \mathrm{s})$ & $34.34 \pm 2.38$ & $33.28 \pm 6.12$ & $34.27 \pm 5.98$ & 0.270 & NA & NA & NA \\
\hline $\operatorname{EDV}(\mathrm{mm} / \mathrm{s})$ & $30.97 \pm 2.31$ & $30.51 \pm 3.62$ & $30.76 \pm 4.18$ & 0.636 & NA & NA & NA \\
\hline RI & $0.81 \pm 0.24$ & $0.72 \pm 0.17$ & $0.69 \pm 0.25$ & 0.002 & 0.009 & 0.807 & 0.008 \\
\hline
\end{tabular}

CAA, coronary artery aneurysm; CAE, coronary artery ectasia; NCA, normal coronary artery; baPWV, brachial-ankle pulse wave rate; ABI, ankle-brachial index; LDL/HDL, low-density lipoprotein/high-density lipoprotein; IMT, intima-media thickness; PCV, peak systolic velocity; EDV, end-diastolic velocity; RI, resistance index; NA, not applicable.

Table 5 Comparison of concomitant coronary stenosis between the CAA and CAE groups

\begin{tabular}{lccc}
\hline Coronary stenosis indexes & CAA $(n=100)$ & CAE $(n=100)$ & P \\
\hline Gensini score & $81.9 \pm 30.1$ & $37.5 \pm 19.7$ & $<0.001$ \\
SYNTAX score & $10.3 \pm 6.6$ & $5.6 \pm 4.0$ & $<0.001$ \\
Concomitant stenosis $(\%)$ & 75 & 43 & $<0.001$ \\
\hline
\end{tabular}

CAA, coronary artery aneurysm; CAE, coronary artery ectasia.

$1.16 \pm 0.48 \mathrm{RU} / \mathrm{mL}, \mathrm{P}=0.005)$.

The MPO level was significantly higher in the CAE group $(0.84 \pm 0.25 \mathrm{ng} / \mathrm{mL})$ than in the CAA group $(0.73 \pm 0.27 \mathrm{ng} / \mathrm{mL})$ and the NCA group $(0.69 \pm 0.36 \mathrm{ng} / \mathrm{mL})$ $(\mathrm{P}=0.002)$. The GDF-15 level $(352.20 \pm 105.13 \mathrm{ng} / \mathrm{L})$ was significantly higher in the $\mathrm{CAE}$ group than in the CAA group $(323.78 \pm 57.75 \mathrm{ng} / \mathrm{L})$ and NCA group $(308.90 \pm 98.54 \mathrm{ng} / \mathrm{L})$ $(\mathrm{P}=0.003)$.

\section{Comparison of atherosclerotic indexes between the CAA and CAE groups}

Table 4 shows that there is no significant difference in baPWV values among the three groups. The ABI values in the CAA and CAE groups were significantly lower than that in the NCA group $(0.78 \pm 0.19$ vs. $0.79 \pm 0.20$ vs. $0.85 \pm 0.18$, $\mathrm{P}=0.017)$. The rate of low-density lipoprotein (LDL)/highdensity lipoprotein (HDL) was significantly higher in the CAA group than in the NCA group $(2.73 \pm 1.29$ vs. $2.33 \pm 0.83$, $\mathrm{P}=0.030$ ).

The results of carotid ultrasonography (PSV and EDV) were not significantly different among the three groups. IMT was significantly higher in the CAA group than in the CAE and NCA groups $(0.89 \pm 0.38$ vs. $0.78 \pm 0.24 v s$. $0.76 \pm 0.37 \mathrm{~mm}, \mathrm{P}=0.019)$. The $\mathrm{RI}$ value was also significantly higher in the CAA group $(0.81 \pm 0.24)$ than in the CAE group $(0.72 \pm 0.17)$ and NCA group $(0.69 \pm 0.25)(\mathrm{P}=0.002)$.

A total of 75 patients in the CAA group, and 43 patients in the CAE group displayed coexistence with coronary stenosis (Table 5). The percentage of patients with concomitant stenosis was significantly higher in the CAA group than in the CAE group $(\mathrm{P}<0.001)$. The Gensini score was significantly higher in the CAA group than in the CAE group $(81.9 \pm 30.1$ vs. $37.5 \pm 19.7, \mathrm{P}<0.001)$. The SYNTAX score was also significantly higher in the CAA group than in the CAE group $(10.3 \pm 6.6$ vs. 5.6 $44.0, \mathrm{P}<0.001)$.

\section{Comparison of platelet activation and aggregation between the $C A A$ and $C A E$ groups}

Table 6 shows that there is no significant difference in the vWF value among the three groups. Values for CD62P 
Table 6 Comparison of platelet activation and aggregation among the CAA, CAE, and NCA groups

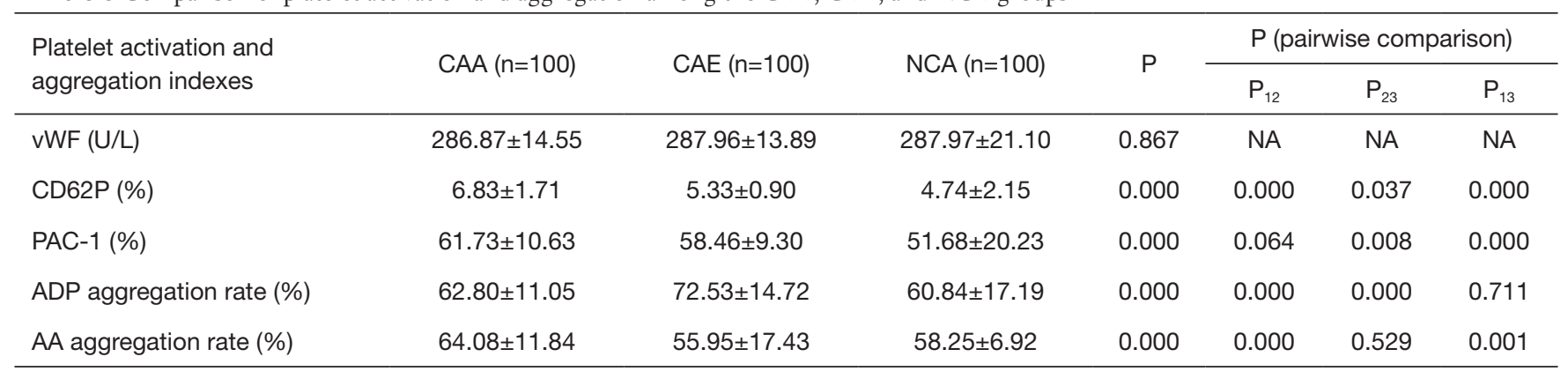

CAA, coronary artery aneurysm; CAE, coronary artery ectasia; NCA, normal coronary artery; vWF, von Willebrand factor; PAC-1, procaspase-activating compound 1; ADP, adenosine 5'-diphosphate; AA, arachidonic acid; NA, not applicable.

Table 7 Comparison of thromboelastography indexes among the CAA, CAE, and NCA groups

\begin{tabular}{|c|c|c|c|c|c|c|c|}
\hline $\begin{array}{l}\text { Thromboelastography } \\
\text { indexes }\end{array}$ & CAA $(n=100)$ & CAE $(n=100)$ & $\operatorname{NCA}(n=100)$ & $P$ & \multicolumn{3}{|c|}{$\mathrm{P}$ (pairwise comparison) } \\
\hline $\mathrm{K}(\mathrm{s})$ & $81.45 \pm 5.83$ & $79.77 \pm 9.07$ & $83.10 \pm 4.93$ & 0.003 & 0.321 & 0.005 & 0.093 \\
\hline$\alpha$ angle $\left({ }^{\circ}\right)$ & $70.37 \pm 11.96$ & $74.25 \pm 12.47$ & $67.39 \pm 19.20$ & 0.005 & 0.076 & 0.009 & 0.468 \\
\hline $\mathrm{MA}(\mathrm{mm})$ & $68.30 \pm 3.75$ & $69.60 \pm 5.29$ & $69.24 \pm 4.66$ & 0.125 & NA & NA & NA \\
\hline
\end{tabular}

CAA, coronary artery aneurysm; CAE, coronary artery ectasia; NCA, normal coronary artery; K, thrombin formation time; $\alpha$ angle, fibrin clot formation rate; MA, blood clot strength; $R$, reaction time; NA, not applicable.

$(6.83 \% \pm 1.71 \%$ vs. $4.74 \% \pm 2.15 \%, \mathrm{P}=0.000), \mathrm{PAC}-1$ $(61.73 \% \pm 10.63 \%$ vs. $51.68 \% \pm 20.23 \%, \mathrm{P}=0.000)$, and the AA aggregation rate $(64.08 \% \pm 11.84 \%$ vs. $58.25 \% \pm 6.92 \%$, $\mathrm{P}=0.001)$ were significantly higher in the CAA group than in the NCA group. Values for CD62P $(5.33 \% \pm 0.90 \% v s$. $4.74 \% \pm 2.15 \%, \mathrm{P}=0.037)$, PAC $-1(558.46 \% \pm 9.30 \%$ vs. $51.68 \% \pm 20.23 \%, \mathrm{P}=0.008)$, and the ADP aggregation rate $(72.53 \% \pm 14.72 \%$ vs. $60.84 \% \pm 17.19 \%, \mathrm{P}=0.000)$ were significantly higher in the CAE group than in the NCA group.

The CD62P value $(6.83 \% \pm 1.71 \%$ vs. $5.33 \% \pm 0.90 \%$, $\mathrm{P}=0.000)$ and the $\mathrm{AA}$ aggregation rate $(64.08 \% \pm 11.84 \%$ vs. $55.95 \% \pm 17.43 \%, \mathrm{P}=0.000$ ) were significantly higher in the CAA group than in the CAE group. The ADP aggregation rate was significantly higher in the CAE group than in the CAA group $(72.53 \% \pm 14.72 \%$ vs. $62.8 \% \pm 11.05 \%, \mathrm{P}=0.000)$.

The TEG results in Table 7 show that the $\mathrm{K}$ value $(79.77 \pm 9.07$ vs. $83.10 \pm 4.93 \mathrm{~s}, \mathrm{P}=0.005)$ and $\mathrm{R}$ value $(7.71 \pm 1.28$ vs. $8.81 \pm 3.10 \mathrm{~min}, \mathrm{P}=0.004)$ were significantly lower in the CAE group than in the NCA group, whereas the $\alpha$ angle was significantly higher in the CAE group than in the NCA group $\left(74.25^{\circ} \pm 12.47^{\circ}\right.$ vs. $67.39^{\circ} \pm 19.20^{\circ}$,
$\mathrm{P}=0.009)$. There were no significant differences in the $\mathrm{K}$ value, $\alpha$ angle, and $\mathrm{R}$ value between the CAA and NCA groups. There was no significant difference in MA among the three groups.

\section{Discussion}

The incidence of coronary artery dilation is lower than that of CAD. Previous studies have demonstrated that atherosclerosis may be a potential cause of coronary artery dilatation, as many dilatations have been observed concomitantly with CAD (10-12). Other observations have revealed that coronary artery dilatation is also found in many patients without significant atherosclerotic stenosis, and that inflammation may be a potential cause of dilatation $(13,14)$. Because of the small number of cases, previous studies did not classify coronary artery dilatation into CAA and CAE. In our previous study, we divided coronary artery dilatation into CAA and CAE, and systematically compared the differences in angiographic characteristics and cardiovascular risk factors between the two; we found that there may be a closer relationship between CAA and 
atherosclerosis (7). We further clarified that the incidence of CAE is closely related to inflammation, whereas the incidence of CAA is closely related to atherosclerosis.

Previous autopsy studies have confirmed that destruction of the extracellular matrix in the middle layer of blood vessels is the main pathological feature of coronary artery dilatation, especially the destruction and degradation of elastic fibers (15). Therefore, various types of proteolytic enzymes, particularly MMP, have received increasingly more attention. Lamblin et al. found that the detection rate of MMP-3 in coronary artery dilatation was significantly increased. It was speculated that the proteolysis mediated by MMP caused the degradation of the middle layer of the coronary wall, thus playing an important role in the formation of coronary artery dilatation (16). Some studies have shown that MMP-9 is overexpressed in coronary artery dilatation and is related to the severity of coronary artery dilatation $(17,18)$. Proteolytic enzymes, such as serine proteases and thiol proteases, are also related to elastic fiber degradation, which has not been studied in coronary artery dilatation. Aside from proteolytic enzymes, increased vasodilation factors, inflammatory activation products, and endothelial injury promote inflammation and regulate the secretion of proteolytic enzymes, which affect the progression of coronary artery dilatation. The concentration of nitric oxide (NO), a classic diastolic factor in cardiovascular disease studies, has been shown to decrease in many CAD studies but increase in coronary artery dilatation studies. $\mathrm{NO}$ can directly stimulate vasodilation and damage the vascular endothelium, thereby promoting inflammation (19,20). Turhan et al. (21) and Yilmaz et al. (22) found that E-selectin, intercellular adhesion molecule-1, and vascular cell adhesion molecule-1 levels were elevated in coronary artery dilatation, suggesting that the increase of adhesion molecules facilitates the adhesion and migration of neutrophils and monocytes to the vascular endothelium, which further promotes inflammation. Moreover, there are abnormal increases of various cytokines and inflammatory cells in coronary artery dilatation. CRP $(23,24), \mathrm{TNF}-\alpha(25)$, IL-6 (26), and NLR (27) all play a role in the occurrence and development of coronary artery dilatation, which can up-regulate the level of MMP, facilitate the expression of adhesion molecules, and promote the progression of inflammation, thus affecting the formation and severity of coronary artery dilatation. The present study's results showed that TNF- $\alpha$, MMP-9, MPO, p-ANCA, c-ANCA, ET-1, and GDF-15 levels were significantly increased in the CAE group, but there was no increase of these indexes in the CAA group, except TNF- $\alpha$, suggesting that inflammatory activation is more important in the incidence of CAE. The vascular inflammatory response in CAE was obvious. This promoted the increase of MMP9, degranulation and release of MPO, and secretion of various hydrolyzed proteins, resulting in damage to elastic fibers and the structure of the blood vessel wall, noticeable thinning of the blood vessel wall, degradation of the extracellular matrix, and injury of the vascular media. Furthermore, inflammation led to the increase of vascular endothelial permeability, increase of ET-1 secretion due to vascular endothelial injury, and increase of endothelial repair factor GDF-15, which had gradually expanded and restructured under the action of mechanical stress, eventually leading to CAE. Conversely, the inflammatory response was limited in CAA, with an increase of only the TNF- $\alpha$ level. The effect of TNF- $\alpha$ is dose-dependent; is autocrine or paracrine at a low dose and does not produce an extensive vascular inflammatory response $(28,29)$. We found no difference in the hsCRP and IL-6 levels between the CAE, CAA, and NCA groups. However, to the best of our knowledge, this study is the first to reveal differences in CAE and CAA. Though, the study included a small sample size, and was conducted at only one center. Also, it is important to point out that different inflammation factors have various functions. Further study may reveal more information about the roles played by the different types of inflammation factors.

It has been suggested that coronary artery dilatation seems to be as closely related to atherosclerosis as coronary stenosis $(30,31)$. There are atherosclerotic changes in coronary dilated blood vessels under the microscope, with lipid fragments containing the cholesterol core and fibrous cap, as well as focal destruction of the middle layer of blood vessels, suggesting that the occurrence of dilatation is related to atherosclerosis (30). A study on blood lipids has shown that the LDL/HDL level was abnormally elevated in coronary artery dilation and is related to its severity (32), revealing that LDL is closely correlated with matrix degradation. Macrophages and smooth muscle cells phagocytize lipids and LDL, and differentiate into foam cells that can damage the endothelium, stimulate the degradation of extracellular matrix, and cause the dilation of atherosclerotic sites (33). Celik et al. implied that carotid intima-media thickening was significantly higher in patients with coronary artery dilation than in the healthy population, suggesting that its pathogenesis may be similar to that of CAD (34). On the contrary, other studies have produced 
completely opposite results. Yetkin et al.'s study (35) showed that carotid artery thickening was significantly lower in patients with coronary artery dilation than in those with CAD. Yetkin and Waltenberger's study (36) also implied that among the risk factors for coronary artery dilation, smoking was significantly increased, while the history of diabetes was significantly decreased for those with $\mathrm{CAD}$, suggesting that there are similarities and differences in atherosclerosis between coronary artery dilation and CAD. Virmani et al. (15) found that patients with coronary artery dilatation concomitant with CAD had more limited segmental dilatation and were more likely to develop a thrombus than those without CAD, suggesting that local dilatation may be more closely related to atherosclerosis. Herein, ABI was significantly lower in the CAA group than in the NCA group, while LDL/HDL was significantly higher in the CAA group than in the NCA group. Carotid ultrasonography indexes (IMT and RI), percentage of patients with concomitant coronary stenosis, Gensini score, and SYNTAX score were significantly higher in the CAA group than in the CAE group, suggesting that the dilatation in CAA was closely related to coronary atherosclerosis. With reference to previous research results, it has been speculated that atherosclerosis leads to local dilatation, slow blood flow, and endothelial cell damage, but the vascular inflammatory response and proteolytic enzyme secretion are not obvious; therefore, only local dilatation of atherosclerotic lesions occurs, resulting in different dilatation characteristics of CAA and CAE.

Importantly, platelets are the only blood components that act on the coagulation system and the inflammatory system, which play a vital role in the development of coronary artery dilatation. Many studies have shown that platelet activation products $\beta$-TG and CD62P are significantly higher in coronary artery dilatation than in NCA, suggesting the presence of platelet activation in coronary artery dilatation, which is considered to be related to slow blood flow and local blood turbulence during coronary artery dilatation $(37,38)$. Vascular endothelial injury increased endothelial permeability, and collagen exposure lead to platelet activation and aggregation. Activated platelets stimulate the blood vessel wall, exacerbating plaque formation and thrombosis at dilated sites. Activated platelets also promote vascular inflammation, between which the interaction results in a coagulation-anticoagulation imbalance that initiates a coagulation cascade, thereby causing coagulation. Platelet activation plays an important role in the formation of thrombosis and the progression of chronic inflammation in coronary artery dilation $(39,40)$. In the present study, the CD62P level, PAC-1 level, TEG indexes, and the ADP aggregation rate were significantly higher in the CAE group than in the NCA group, which confirmed that there was extensive platelet activation in CAE. Moreover, the CD62P level, PAC-1 level, and AA aggregation rate were higher in the CAA group than in the NCA group, suggesting that platelet activation was also present in CAA. Based on our conjecture, the increase of different activation indexes indicates that there may be different paths of platelet activation between the CAA and CAE groups. Platelet activation in CAE may be related to the interaction between inflammation and platelets, whereas platelet activation in CAA may be more closely related to atherosclerosis and endothelial injury. Long-term antiplatelet therapy is needed to prevent thrombosis regardless of whether the patient has CAE or CAA.

Many questions remain unanswered by the present study, such as whether anti-inflammatory therapy is beneficial to patients with CAE, and why atherosclerosis manifests as coronary dilatation in some patients but coronary stenosis in many patients. The current research is limited to only clinical research. Further molecular biological research and the establishment of animal models of coronary artery dilatation are needed to explore the pathogenesis of CAA and CAE. Notably, some genomic studies have suggested that coronary artery dilatation might be related to a variation of chromosome 9p21.3, and that this gene may be related to the angiotensin-converting enzyme DD polymorphism, which possibly mediates vascular remodeling (41). These findings will be explored in further studies.

In conclusion, there are close relationships between CAE and inflammation as well as between CAA and atherosclerosis. Antiplatelet therapy is a requisite for the treatment of CAA and CAE because they are prone to platelet activation.

\section{Acknowledgments}

Funding: This study was supported by grants from the Science Foundation of Shanghai Municipal Health Commission (201840112), National Natural Science Foundation of China (81900260), and the Top-level Clinical Project of Shanghai Pudong (PWYgf2018-02).

\section{Footnote}

Reporting Checklist: The authors have completed the 
STROBE reporting checklist. Available at http://dx.doi. org/10.21037/jtd-20-1579

Data Sharing Statement: Available at http://dx.doi. org/10.21037/jtd-20-1579

Peer Review File: Available at http://dx.doi.org/10.21037/jtd20-1579

Conflicts of Interest: All authors have completed the ICMJE uniform disclosure form (available at http://dx.doi. org/10.21037/jtd-20-1579). The authors have no conflicts of interest to declare.

Ethical Statement: The authors are accountable for all aspects of the work in ensuring that questions related to the accuracy or integrity of any part of the work are appropriately investigated and resolved. The study was approved by the Ethics Committee of Shanghai East Hospital Tongji University \{No. [2018]009\} and was conducted in line with the Declaration of Helsinki (as revised in 2013). All patients signed the written informed consent form.

Open Access Statement: This is an Open Access article distributed in accordance with the Creative Commons Attribution-NonCommercial-NoDerivs 4.0 International License (CC BY-NC-ND 4.0), which permits the noncommercial replication and distribution of the article with the strict proviso that no changes or edits are made and the original work is properly cited (including links to both the formal publication through the relevant DOI and the license). See: https://creativecommons.org/licenses/by-nc-nd/4.0/.

\section{References}

1. Jarcho S. Bougon on coronary aneurysm (1812). Am J Cardiol 1969;24:551-3.

2. Trinidad S, Grayzel DM, Rapoport P. Aneurysm of the coronary artery. Ann Intern Med 1953;39:1117-24.

3. Almansori MA, Elsayed HA. Coronary artery ectasia-A sample from Saudi Arabia. J Saudi Heart Assoc 2015;27:160-3.

4. Mavrogeni S, Markousis-Mavrogenis G, Kolovou G. Contribution of cardiovascular magnetic resonance in the evaluation of coronary arteries. World J Cardiol 2014;6:1060-6.

5. Packard $M$, Wechsler HF. Aneurysm of the coronary arteries. Arch Intern Med (Chic) 1929;43:1-14.
6. Díaz-Zamudio M, Bacilio-Perez U, Herrera-Zarza MC, et al. Coronary artery aneurysms and ectasia: role of coronary CT angiography. Radiographics 2009;29:1939-54.

7. Luo Y, Tang J, Liu X, et al. Coronary artery aneurysm differs from coronary artery ectasia: angiographic characteristics and cardiovascular risk factor analysis in patients referred for coronary angiography. Angiology 2017;68:823-30.

8. Kawsara A, Nunez Gil IJ, Alquahtani F, et al. Management of coronary artery aneurysms. JACC Cardiovasc Interv 2018;11:1211-23.

9. Aboeata AS, Sontineni SP, Alla VM, et al. Coronary artery ectasia: current concepts and interventions. Front Biosci (Elite Ed) 2012;4:300-10.

10. Giannoglou GD, Antoniadis AP, Chatzizisis YS, et al. Prevalence of ectasia in human coronary arteries in patients in northern Greece referred for coronary angiography. Am J Cardiol 2006;98:314-8.

11. Valente S, Lazzeri C, Giglioli C, et al. Clinical expression of coronary artery ectasia. J Cardiovasc Med (Hagerstown) 2007 8:815-20.

12. Swanton RH, Thomas ML, Coltart DJ, et al. Coronary artery ectasia-a variant of occlusive coronary arteriosclerosis. Br Heart J 1978;40:393-400.

13. Li JJ, Nie SP, Qian XW, et al. Chronic inflammatory status in patients with coronary artery ectasia. Cytokine 2009;46:61-4.

14. Li JJ, He JG, Nan JL, et al. Is systemic inflammation responsible for coronary artery ectasia? Int $\mathrm{J}$ Cardiol 2008;130:e69-70.

15. Virmani R, Robinowitz M, Atkinson JB, et al. Acquired coronary arterial aneurysms: an autopsy study of 52 patients. Hum Pathol 1986;17:575-83.

16. Lamblin N, Bauters $C$, Hermant $X$, et al. Polymorphisms in the promoter regions of MMP-2, MMP-3, MMP-9 and MMP-12 genes as determinants of aneurysmal coronary artery disease. J Am Coll Cardiol 2002;40:43-8.

17. Bentzon JF, Pasterkamp G, Falk E. Expansive remodeling is a response of the plaque-related vessel wall in aortic roots of apoE-deficient mice: an experiment of nature. Arterioscler Thromb Vasc Biol 2003;23:257-62.

18. Chatzizisis YS, Coskun AU, Jonas M, et al. Risk stratification of individual coronary lesions using local endothelial shear stress: a new paradigm for managing coronary artery disease. Curr Opin Cardiol 2007;22:552-64.

19. Fukuda S, Hashimoto N, Naritomi H, et al. Prevention of rat cerebral aneurysm formation by inhibition of nitric oxide synthase. Circulation 2000;101:2532-8. 
20. Krüger D, Stierle U, Herrmann G, et al. Exercise-induced myocardial ischemia in isolated coronary artery ectasias and aneurysms ('dilated coronopathy'). J Am Coll Cardiol 1999;34:1461-70.

21. Turhan H, Erbay AR, Yasar AS, et al. Plasma soluble adhesion molecules; intercellular adhesion molecule-1, vascular cell adhesion molecule-1 and E-selectin levels in patients with isolated coronary artery ectasia. Coron Artery Dis 2005;16:45-50.

22. Yilmaz H, Tayyareci G, Sayar N, et al. Plasma soluble adhesion molecule levels in coronary artery ectasia. Cardiology 2006;105:176-81.

23. Turhan H, Erbay AR, Yasar AS, et al. Comparison of C-reactive protein levels in patients with coronary artery ectasia versus patients with obstructive coronary artery disease. Am J Cardiol 2004;94:1303-6.

24. Collins MJ, Borges AJ, Singh G, et al. A giant coronary artery aneurysm in the right coronary artery. Cardiovasc Pathol 2006;15:150-2.

25. Burgner D, Davila S, Breunis WB, et al. A genome-wide association study identifies novel and functionally related susceptibility Loci for Kawasaki disease. PLoS Genet 2009; 5:e1000319.

26. Triantafyllis AS, Kalogeropoulos AS, Rigopoulos AG, et al. Coronary artery ectasia and inflammatory cytokines: link with a predominant Th-2 immune response? Cytokine 2013;64:427-32

27. Sarli B, Baktir AO, Saglam H, et al. Neutrophil-tolymphocyte ratio is associated with severity of coronary artery ectasia. Angiology 2014;65:147-51.

28. Kapadia S, Torre-Amione G, Yokoyama T, et al. Soluble TNF binding proteins modulate the negative inotropic properties of TNF-alpha in vitro. Am J Physiol 1995;268:H517-H525.

29. Van Zee KJ, Kohno T, Fisher E, et al. Tumor necrosis factor soluble receptors circulate during experimental and clinical inflammation and protect against executive tumor necrosis factor-alpha in vitro and in vivo. Proc Natl Acad
Sci U S A 1992,89:4845-9.

30. Demopoulos VP, Olympios CD, Fakiolas CN, et al. The natural history of aneurysmal coronary artery disease. Heart 1997;78:136-41.

31. Ovalı C, Morrad B. Associations between coronary artery disease, aneurysm and ectasia. Kardiochir Torakochirurgia Pol 2017;14:158-63.

32. Kundi H, Gok M, Kiziltunc E, et al. Relation between monocyte to high-density lipoprotein cholesterol ratio with presence and severity of isolated coronary artery ectasia. Am J Cardiol 2015;116:1685-9.

33. Antoniadis AP, Chatzizisis YS, Giannoglou GD. Pathogenetic mechanisms of coronary ectasia. Int J Cardiol 2008;130:335-43.

34. Celik S, Erdogan T, Kasap H, et al. Carotid intima-media thickness in patients with isolated coronary artery ectasia. Atherosclerosis 2007;190:385-7.

35. Yetkin E, Acikgoz N, Aksoy Y, et al. Decreased carotid intima-media thickness in patients with coronary artery ectasia compared with patients with coronary artery disease. Coron Artery Dis 2005;16:495-8.

36. Yetkin E, Waltenberger J. Novel insights into an old controversy: is coronary artery ectasia a variant of coronary atherosclerosis? Clin Res Cardiol 2007;96:331-9.

37. Yasar AS, Erbay AR, Ayaz S, et al. Increased platelet activity in patients with isolated coronary artery ectasia. Coron Artery Dis 2007;18:451-4.

38. O'Connor CM, Gurbel PA, Serebruany VL. Usefulness of soluble and surface-bound $\mathrm{P}$-selectin in detecting heightened platelet activity in patients with congestive heart failure. Am J Cardiol 1999;83:1345-9.

39. Rondina MT, Weyrich AS, Zimmerman GA. Platelets as cellular effectors of inflammation in vascular diseases. Circ Res 2013;112:1506-19.

40. Wagner DD, Burger PC. Platelets in inflammation and thrombosis. Arterioscler Thromb Vasc Biol 2003;23:2131-7.

41. Ozaki K, Tanaka T. Molecular genetics of coronary artery disease. J Hum Genet 2016;61:71-7.
Cite this article as: Wei W, Wang $\mathrm{X}$, Huang Z, Li X, Luo Y. Difference in inflammation, atherosclerosis, and platelet activation between coronary artery aneurysm and coronary artery ectasia. J Thorac Dis 2020;12(10):5811-5821. doi: $10.21037 /$ jtd-20-1579 